RUNNING HEAD: Brightness of Affective Pictures

Brightness Differences Influence the Evaluation of Affective Pictures

\author{
Daniël Lakens \\ Eindhoven University of Technology \\ Daniel A. Fockenberg \\ VU University Amsterdam \\ Karin P. H. Lemmens, Jaap Ham, \& Cees J. H. Midden \\ Eindhoven University of Technology
}

In press, Cognition \& Emotion

7588 words

KEYWORDS: affective pictures, emotions, brightness bias, IAPS, color psychology.

Author Note:

We thank Dik Hermes for valuable assistance with MATLAB programming.

Correspondence can be addressed to Daniël Lakens, Human Technology Interaction Group, IPO 1.24, PO Box 513, 5600MB Eindhoven, The Netherlands. E-mail: D.Lakens@tue.nl. 


\begin{abstract}
We explored the possibility of a general brightness bias: brighter pictures are evaluated more positively, while darker pictures are evaluated more negatively. In Study 1 we found that positive pictures are brighter than negative pictures in two affective picture databases (the IAPS and the GAPED). Study 2 revealed that because researchers select affective pictures on the extremity of their affective rating without controlling for brightness differences, pictures used in positive conditions of experiments were on average brighter than those used in negative conditions. Going beyond correlational support for our hypothesis, Studies 3 and 4 showed that brighter versions of neutral pictures were evaluated more positively than darker versions of the same picture. Study 5 revealed that people categorized positive words more quickly than negative words after a bright prime picture, and vice versa for negative pictures. Together, these studies provide strong support for the hypotheses that picture brightness influences evaluations.
\end{abstract}




\section{Brightness Differences Influence the Evaluation of Affective Pictures}

Whether a picture is judged to be positive or negative is obviously determined by the content of the picture. A picture of two puppies on a sunny meadow will be liked more than a picture of snake crawling around in the dark. What might be less obvious is the possibility that basic perceptual characteristics of the picture can also influence whether the picture is evaluated more or less positively, independent of its content. Would the picture of the two puppies be evaluated somewhat less positively if the picture was overall darker, and would the picture of the snake be liked somewhat better if it was overall brighter?

Evaluative information is one of the most important conceptual dimensions of meaning (Osgood, Succi, \& Tannenbaum, 1957). Not surprisingly, research suggests that people may spontaneously evaluate all kinds of environmental objects, such as words, pictures, sounds, and odors (e.g., Klauer \& Musch, 2003). Likewise, brightness is one of the most important sources of perceptual information, as brightness differences provide contrast, which is essential for vision (Valberg, 2005). Cross-cultural research has shown that people have a strong association between valence and brightness (Adams \& Osgood, 1973). Specifically, people tend to evaluate brighter colors more positively than darker colors (Guilford, 1934; Valdez \& Mehrabian, 1994). Given that the preference for brighter colors is also observed in monkeys (Humphrey, 1971), the association between valence and brightness is suggested to reflect a general evolutionary predisposition of diurnal animals, for which darkness increases anxiety and negative feelings (Grillon, Pellowski, Merikangas, \& Davis, 1997).

Despite the fact that brightness and valence information is ubiquitous and important in daily life, there is surprisingly little research that has examined the consequences of the brightness-valence association for naturalistic stimuli such as real-life photographs. However, recent theoretical developments suggest the possibility that the brightness of pictures should 
influence how they are evaluated. In particular, research on embodied cognition highlights how concrete information can influence the way people think about more abstract concepts (Barsalou, 1999). For example, the weight of a clipboard can influence the answers people provide on a questionnaire because of mental associations between weight and importance (Jostmann, Lakens, \& Schubert, 2009; see also Ackerman, Nocera, \& Bargh, 2010; Chandler, Reinhard, \& Schwarz, 2012; Maglio, \& Trope, 2012; Schneider, Rutjens, Jostmann, \& Lakens, 2011; Stewart, McVeigh, Stojkov, \& Daubman, 2012).

Work on color psychology (Elliot \& Maier, 2007; 2012) has shown that colors carry meaning, and concrete color cues can influence behavior, cognition, and emotions. For example, the color red influences how attractive someone of the opposite sex is judged to be (Elliot et al., 2010; Elliot \& Niesta, 2008; Pazda, Elliot, \& Greitemeyer, 2012). In the domain of brightness, the color of the uniform worn by sport teams has been shown to influence perceived aggressiveness, with teams dressed in black being penalized more often than teams wearing non-black uniforms (Frank \& Gilovich, 1988). In addition, brightness is associated with valence (e.g., Lakens, Semin, \& Foroni, 2012; Meier, Robinson, \& Clore, 2004; Okubo \& Ishikawa, 2011) and morality (Sherman \& Clore, 2009).

Based on these recent insights, it is conceivable that brighter pictures are evaluated more positively, irrespective of the content of the picture. In the current research, we explored the possibility of such a general brightness bias: brighter pictures are evaluated more positively, while darker pictures are evaluated more negatively. Naturalistic photographs that differ in valence are often used to induce emotions, not only in daily life (e.g., in advertisements, see Mitchell, 1986), but also to induce emotions in a controlled manner in experimental research. If brightness influences how pictures are evaluated, such a brightness bias could be widely applied in every domain where visual stimuli are used to communicate 
affective information. At the same time, this brightness bias would be relevant for emotion researchers who rely on affective pictures to experimentally manipulate valence.

\section{Overview of the current research}

The aim of the current investigation was to determine whether the brightness of naturalistic photographs influences how these photographs are evaluated. First, we report an analysis of the relationship between valence ratings and brightness values in two affective pictures databases, both by calculating correlations across the entire databases, as well as more detailed analyses of specific subsets of the pictures. Note that such a correlation between the valence and brightness in these databases is only problematic, if researchers fail to select equally bright positive and negative stimuli for their experiments. Therefore, in Study 2 we investigated whether brightness differences in positive and negative pictures are indeed present in the pictures researchers select from the IAPS for their experiments. Specifically, we examined 47 studies in which IAPS pictures were used, and compared the average brightness values of the positive, neutral, and negative pictures used as affective stimuli.

We conducted three empirical studies to provide experimental support for the hypothesized brightness bias. In the first two experiments (Studies 3 and 4), we manipulated the brightness of photographs, and investigated whether this brightness manipulation has an influence on how people evaluate the stimuli. Finally, in Study 5 we used a lighter and a darker version of the same picture as a prime in an affective priming task, to examine whether the brightness of pictures influences the speed with which people evaluate subsequent affective stimuli.

\section{Study 1: Valence and Brightness in Picture Databases (IAPS and GAPED)}

The International Affective Picture System (IAPS; Lang, et al., 2008) is one of the oldest and most widely used database of affective pictures in psychology. It consists of natural pictures of a wide range of topics for which valence, arousal and dominance ratings are 
available. Recently, the Geneva Affective Picture Database (GAPED; Dan-Glauser \& Scherer, 2011) has been presented. This database consists of negative pictures (depicting snakes, spiders, animal mistreatments, human concerns), neutral pictures, and positive pictures, for which valence, arousal, and normative ratings are available. Because the pictures in these databases consist of naturalistic photographs, they do not only differ in their valence, but also in perceptual characteristics such as brightness, contrast, and spatial frequency. Therefore, these widely used picture databases provide a perfect first testing ground to examine the brightness bias.

The developers of the IAPS have recommended researchers to control for perceptual differences of the pictures (e.g., Bradley \& Lang, 2007). However, although some studies carefully control for differences in some perceptual characteristics of the stimuli such as brightness and contrast (e.g., Calvo \& Nummenmaa, 2007; De Cesarei \& Codispoti, 2006), naturalistic pictures can differ on many perceptual characteristics, and it is practically impossible to always control for all possible differences between conditions. In addition, there is no empirical research that provides a clear indication that the evaluations of affective pictures are influenced by perceptual stimulus characteristics. Only one study has raised the possibility that researchers might select specific thematic subsets of pictures that differ both in valence as in spatial frequency, although no systematic relationship was observed between spatial frequencies and valence ratings across the entire database (Delplanque, N'diaye, Scherer, \& Grandjean, 2007). Furthermore, even if researchers would want to control for perceptual characteristics, information about the perceptual characteristics of affective pictures (e.g., the brightness value associated with a specific picture) is not readily available, and researchers might not know how to determine these values.

We analyzed the relation between the valence and brightness values of affective pictures of the most recent version of the IAPS (Lang, et al., 2008) and the GAPED (Dan- 
Glauser \& Scherer, 2011). The IAPS has been used extensively in previous research, and the GAPED seems to be an equally useful tool for future research. The relationship between the valence and brightness values of affective pictures in these databases was analyzed for the databases as a whole, and for specific subsets of the stimuli.

Brightness values. The brightness of the affective pictures was determined using MATLAB software version R2012b. Average picture brightness is calculated from the brightness of each pixel of the picture, and is represented by a value ranging from 0 (completely black) to 255 (completely white). For each picture, the RGB pixels are converted to brightness values using an arithmetic mean model $V=(R+G+B) / 3$, following Bradley, Hamby, Löw, and Lang (2007). The brightness values calculated by using alternative functions, such as the weighted RGB conversion function for video luma $\left(\mathrm{Y}^{\prime}=0.299 \mathrm{R}+\right.$ $0.587 \mathrm{G}+0.114 \mathrm{~B})$ or CIE Luminance $\left(\mathrm{Y}_{709}=0.2126 \mathrm{R}+0.7152 \mathrm{G}+0.0722 \mathrm{~B}\right)$ were almost perfectly correlated with the arithmetic mean model (all $r$ 's $>.99$ ). Note that these brightness values are based on characteristics of the stimuli, and do not take additional sources of brightness variations into account, such as differences in the brightness settings of computer monitors. The current studies are focused on relative brightness differences within a stimulus set, but researchers should be aware that monitor settings and environmental lighting conditions can provide an additional source of variation between participants.

In addition to brightness, contrast is the most basic characteristic of an image. Following Peli (1990), we have calculated the root mean square (RMS) contrast, which is defined as the standard deviation of the intensity of all pixels in a picture, for all IAPS and GAPED stimuli. To enable researchers to easily check the brightness values of affective pictures in the IAPS and GAPED databases, two files are available in the supplementary materials with the average brightness and contrast values for each picture in the IAPS and GAPED. 
Brightness-valence correlations. The brightness of the 1182 IAPS pictures was positively correlated with their valence (as provided by Lang et al., 2008) ${ }^{1}, r=.063, p=.031$. Brightness of IAPS pictures was not related to arousal or dominance judgments, $r=-.033, p=$ .252 and $r=.037, p=.257$, respectively (see Figure 1 ). The brightness values for the 730 GAPED pictures were positively correlated with their evaluations (as provided by DanGlauser \& Scherer, 2011), $r=.105, p=.005$ (see Figure 1). Brightness of the GAPED pictures was negatively related to the arousal values of the pictures, $r=-.208, p<.001$, which is most likely due to the strong negative correlation between the valence and arousal ratings in the GAPED, $r=-.853, p<.001$. In both affective picture sets, contrast and brightness are significantly correlated (for the IAPS, $r=.198, p<.001$, for the GAPED, $r=.090, p=.016$ ). Importantly however, contrast did not correlate with the evaluations of the affective pictures (for the IAPS, $r=-0.024, p<.402$, for the GAPED, $r=-0.056, p=.128$ ). When controlling for RMS contrast, the relationship between valence and brightness in the GAPED $(r=.111, p=$ $.003)$ and IAPS ( $r=.069, p=.018)$ was not substantially affected.

Average brightness differences for specific subsets. The GAPED consists of six specific categories, namely positive, neutral, and four types of negative pictures (snakes, spiders, animal mistreatments, and human concerns). Brightness differences between these specific subsets were analyzed with an ANOVA with category as the only factor. As expected, average brightness of the stimuli differed significantly between categories, $F(5,324.78)=$ 7.94, $p<.001, \eta_{\mathrm{p}}{ }^{2}=.05$ (Welch's $F$ statistics are reported for GAPED pictures due to a violation of homogeneity of variance for their brightness values). Planned comparisons reveal that both neutral and positive pictures were brighter than negative pictures, $t(120.04)=5.04, p$ $<.001$, and $t(156.66)=2.64, p=.009$, respectively, whereas positive and neutral pictures did not differ in brightness, $t(209.68)=1.62, p=.11$ (see Table 1 for average brightness values). 
For the IAPS, no subsets have been specified. That is, although some pictures can be related to specific positive or negative emotions (Mikels, Fredrickson, Larkin, Lindberg, Maglio, \& Reuter-Lorenz, 2005), there are no formal guidelines on how all pictures can be grouped into thematic subsets. At the same time, many researchers select thematic subsets from the IAPS, for example focusing on stimuli that depict 'attacking animals and humans', 'erotic pictures', or 'household objects'. Because thematic subsets of the IAPS database have not been specified, two of the authors of the current manuscripts independently assigned all IAPS pictures to thematic subsets. One coder categorized the 1182 pictures into 33 thematic subsets while the second coder categorized the pictures into 27 different subsets. There was initial agreement on 759 pictures (63.58\%). Minor conflicts (e.g., creating a 'snake’ category, merging family or children categories into 'social', merging 'transport' into the 'technology' category, adding 'reptile' and 'insects' to the 'wildlife' category) were resolved by a third author while the remaining pictures were assigned to a category 'other' $(n=44)$. Categories were merged to prevent less than 10 pictures in a single category, with the exception of the 'spider' and 'mushroom' groups. Spiders are often used as negative stimuli and are a separate category in the GAPED, while several publications explicitly mentioned the use of mushrooms as neutral stimuli (e.g., Aue, Flykt, \& Scherer, 2007, Wieser, Mühlberger, Alpers, Macht, Ellgring, \& Pauli, 2006; Yartz \& Hawk, 2001).

The average brightness values of the thematic subsets for the IAPS are reported in Appendix A. The average brightness values for each category were compared with the average brightness value of all IAPS pictures $(M=96.13, S D=39.78)$ with one-sample $t$-tests to determine whether a thematic category differed significantly on brightness. Especially noteworthy are the relatively dark ‘attacking humans or animals’ and 'snake’ categories, which are widely used in research, and the relatively bright 'household objects' category, which is often used as a neutral control category. Note that within each thematic subset the pictures 
often differ widely on valence, and that the positive brightness-valence association holds for several subcategories. Our main conclusion based on these analyses is that selecting IAPS stimuli based on certain specific thematic subsets will potentially introduce brightness differences in the experimental design. This is further supported by an analysis of the brightness values in a One-way ANOVA with category as the only factor, $F(26,1155)=3.82$, $p<.001, \eta_{\mathrm{p}}^{2}=.08$

Discussion. In both the IAPS as GAPED affective picture databases, we observed a clear brightness bias: the brightness value of the pictures was correlated with their valence ratings, with brighter pictures being evaluated more positively. Admittedly, the correlations between valence and brightness in the IAPS and GAPED are rather small. Nevertheless, the fact that the brightness of affective pictures correlates with the explicit evaluation of the images in a carefully selected picture set that contains highly affective images (e.g., death, nudity) supports the robustness of this relationship. Researchers should heed the recommendations by the creators of the IAPS to control for perceptual differences in the selected pictures (Bradley \& Lang, 2007), especially when selecting specific subsets of the IAPS (such as attacking animals or snakes).

As noted previously, researchers typically do not digitally adjust their stimuli to control for brightness differences in the IAPS pictures. However, it is conceivable that instead of digitally adjusting the brightness levels in affective pictures, researchers may take special care to select positive and negative pictures of equal brightness from the database across conditions. In the following study, we therefore examine whether researchers have controlled for brightness differences by carefully selecting equally bright positive and negative stimuli in their experiments.

Study 2: Brightness Values of Affective Pictures Selected as Experimental Stimuli 
We examined the possibility that the positive relationship between the valence and brightness of IAPS pictures would translate into a brightness difference between the positive and negative stimuli selected by researchers as experimental stimuli. If such a brightness difference is observed, it would clearly demonstrate that the recommendation to control for brightness differences when selecting affective pictures deserves more attention from researchers.

Literature search. Studies to be included in the meta-analysis were collected by searching PsychInfo and Google Scholar for articles citing any version of the technical manual of the IAPS (e.g., Lang, et al., 2008). Because the GAPED is a relatively new database, it was not possible to include studies that selected stimuli from the GAPED in the meta-analysis.

Inclusion criteria. We aimed to compare the average brightness values of positive, neutral, and negative IAPS pictures used in previous research. Studies were included in which original IAPS pictures were used, and where two conditions used either positive, negative, or neutral stimuli. If studies subdivided stimuli (e.g., negative low arousal and negative high arousal) brightness values were averaged over all negative, positive, or neutral stimuli. Datasets were included in the meta-analysis when the IAPS numbers were reported in the article, or when upon request authors were kind enough to provide the IAPS numbers of the stimuli they used. Two studies explicitly noted controlling for luminance (Hindi Attar, Anderson, \& Müller, 2010; Sterzer, Stadler, Krebs, Kleinschmidt, \& Poustka, 2005); the remaining studies did not mention stimulus brightness.

Data Analysis. Each publication was treated as a single observation where a researcher freely selected any number of positive, negative, or neutral stimuli from the IAPS. We calculated the average brightness values for the affective pictures researchers selected for each experimental condition. Because the same (group of) researcher(s) selected the stimuli for each experimental condition, the average brightness values for the different experimental conditions 
is treated as a within subjects variable. The articles included in the analysis are marked with an

* in the references, and referred to by the name of the first author and the year of publication in Appendix B. All 47 studies included negative pictures, 45 studies included positive pictures, and 27 studies included neutral pictures.

Results. Data screening identified three studies where the brightness value in one of the conditions was high enough to be considered an outlier (more than 3 SD's above the average brightness value for stimuli from that valence condition $)^{2}$. Because these outliers are mainly due to the low number of stimuli used in the three studies, we excluded these studies from the analysis.

The average brightness values for the stimuli used in the positive, neutral and negative conditions in each study can be found in Appendix B. The IAPS pictures researchers select for positive trials are on average brighter $(M=102.24, S D=11.25)$ than the pictures selected for negative trials $(M=86.56, S D=11.10)$ and neutral trials $(M=91.89, S D=9.88)$. Because not all 44 studies used positive, negative, and neutral conditions, we performed three repeated measures ANOVA's revealing that the average brightness values for the positive, neutral, and negative conditions differed significantly from each other (positive vs. negative, $F(1,41)=$ 32.97, $p<.001, \eta_{\mathrm{p}}{ }^{2}=.45$, positive vs. neutral, $F(1,21)=12.21, p=.002, \eta_{\mathrm{p}}{ }^{2}=.37$, neutral vs. negative, $\left.F(1,23)=7.96, p=.01, \eta_{\mathrm{p}}{ }^{2}=.26\right)$. For a visual representation of the average brightness differences between the positive and negative and neutral conditions, see Figure 2. This brightness difference is most likely the consequence of the fact that researchers select stimuli that are extremely positive and negative, and loosely corresponds to average brightness difference between the 150 most positive and most negative IAPS pictures. Indeed, a closer look at the stimuli that are selected makes clear that the likelihood that stimuli are selected increases with the extremeness of their valence (see Appendix C). 
Discussion. The results of Study 2 are clear-cut: Positive stimuli are on average brighter than negative stimuli in published studies in which researchers selected stimuli from the IAPS database. This brightness difference is a consequence of the positive relationship between stimulus valence and brightness in the IAPS, combined with the fact that researchers do not control for brightness differences. There is enough variability in brightness ratings in the positive and negative extremes of the IAPS database to select positive and negative pictures that do not differ on brightness. Therefore, we suggest that researchers should not select a random sample of positive and negative stimuli, but instead take care to control for brightness differences.

The current investigation has only examined 47 experiments. There are many more studies that have used IAPS pictures, and therefore one may wonder whether the current selection is representative of all studies that have used the IAPS. However, as Study 1 revealed, there is a positive relationship between the valence and brightness of pictures in the IAPS. Because researchers tend to select stimuli from an affective picture database on the extremeness of their evaluative ratings, it seems reasonable to assume that the current results will generalize to all studies that select stimuli from the IAPS database, unless researchers actively control for brightness differences. It should further be noted that the final set of 47 studies came from a wide range of researchers, were published in many different journals, and investigated a wide range of research questions.

It is important to acknowledge the correlational nature of the relationship between the brightness and valence of affective pictures reported so far. There are two possible explanations for the brightness bias in affective pictures observed in Study 1 and 2. First, positive stimuli might be brighter in real life, or are typically encountered in brighter environments. Perhaps fear-inducing animals can only be photographed in dark holes, whereas fluffy young bunnies can only be photographed on sunny spring days. On the other hand, 
people might use brightness as a cue when they evaluate pictures, or when they select affective pictures from a larger database (e.g., the internet). To provide more conclusive support for our assumption that brightness differences can have a causal influence on how stimuli are evaluated, we performed three experiments in which picture brightness is experimentally manipulated, and investigate how these brightness differences influence the evaluation of these pictures.

\section{Study 3: Picture Brightness Influences Relative Evaluations}

In this first experimental investigation of the influence of picture brightness on evaluations, two versions of the same IAPS picture were briefly presented in consecutive order. In half of the trials, the two versions of the pictures differed in brightness. In the other half of the (filler) trials, the pictures differed on unrelated perceptual characteristics (e.g., saturation, vibrancy). Participants were asked to indicate which of the two versions of the pictures they considered more positive. We expected that participants would prefer the brighter of the two pictures in the experimental trials.

\section{Method.}

Participants. Forty-two participants (24 men, mean age 21) were approached through a participant database of the Eindhoven University of Technology and completed the study in return for a monetary compensation of 1 euro.

Stimuli. Ten neutral IAPS pictures were selected as experimental stimuli in the current study (IAPS numbers: 6150, 7000, 7003, 7004, 7010, 7035, 7041, 7045, 7059, 7185). The evaluations of the stimuli as reported by Lang and colleagues (2008) ranged from 4.93 to 5.08 $(M=4.99, S D=0.04)$. The stimuli were selected to cover a wide range of brightness values, with a minimum of 54.65 and a maximum of $176.84(M=110.02, S D=43.95)$. Brightness values were not correlated with valence ratings of the stimuli $(r=-.33, p=.36)$. The brightness of the 10 neutral IAPS pictures was decreased or increased equally (setting the brightness 
adjustment to +40 or -40 for each picture in Adobe Photoshop CS5). Three versions of the same pictures were used in the study: Unchanged pictures (average brightness $=110.02, S D=$ 43.95), brighter pictures (average brightness $=132.78, S D=45.33$ ) and darker pictures (average brightness $=88.23, S D=39.09$ ). The change in brightness was enough to result in a subtle but noticeable difference, without leading to signs of over- or underexposure of the pictures (see Figure 3). A comparison of the differences in contrast between the three conditions did not reach statistical significance, $F(2,27)=2.15, p=.14$. However, with only 10 stimuli in each condition, this test is severely underpowered. The relation between contrast, brightness, and evaluation is examined in more detail in Study 4.

The 10 experimental trials were supplemented by 10 filler trials (IAPS numbers 7012, 7020, 7050, 7055, 7160, 7161, 7179, 7233, 7247, 7950), where an original IAPS picture was presented together with a changed version of the same picture. The changed and original IAPS pictures used as filler trials did not differ in brightness $(M=127.37, S D=39.05$, and $M=$ 127.21, $S D=41.05$, respectively, nor did they differ in contrast, $F$ 's $<1$ ), but they differed in saturation, hue, or vibrancy. These pictures were included to reduce the salience of the brightness differences in the 10 experimental trials.

Procedure. The experiment ostensibly investigated the automatic image editing qualities of different graphic programs. Participants were told they would briefly see two images in a row, and had to indicate which image they judged to be the most positive of the set. It was noted that differences could be subtle, and that there were no right or wrong answers. Each picture was presented against a black background for $1000 \mathrm{~ms}$, with a $200 \mathrm{~ms}$ black screen between the two stimuli. On experimental trials, the unchanged IAPS picture was paired with a brighter version of the same picture on five randomly selected trials, and with a darker picture on the remaining five trials. After the second picture disappeared, participants could choose the first or second picture by pressing one of two buttons on the screen. The 
order in which the two pictures of each set were presented was randomly determined on each trial.

Results. The order in which the pictures were presented did not influence evaluations, and is not further discussed. A one-sample $t$-test against guessing average (5 out of 10) revealed that participants judged the brighter IAPS image to be the most positive picture of the set above guessing average $(M=6.71, S D=2.19), t(41)=5.07, p<.001$. A paired samples $t$ test revealed that participants were more likely to choose the brighter pictures when the unchanged IAPS picture constituted the brighter choice alternative of the pair $(M=3.64, S D=$ 1.32), compared to when the unchanged IAPS picture constituted the darker choice alternative of the pair $(M=3.07, S D=1.31), t(41)=2.52, p=.02$. Importantly however, two one-sample $t$-tests confirmed that the brighter choice alternative was chosen significantly above guessing average both when this meant choosing the unchanged IAPS picture, $t(41)=5.60, p<.001$, as when it meant choosing the IAPS picture that was digitally adjusted in Photoshop, $t(41)=$ $2.82, p=.007$.

Discussion. When participants were asked to indicate which version of the same IAPS picture they found more positive, they choose the brighter version of the same picture above guessing average. This pattern of results was significant both when the unchanged IAPS was the brighter choice alternative, as when the digitally manipulated IAPS picture was the brighter choice alternative. These results provide initial support for the hypothesis that brighter pictures are evaluated more positively, even when picture content is identical. However, preference judgments in the current experiment consisted of a relative preference of the brighter of two pictures. To provide stronger support for the effect of the brightness of pictures on their evaluations, in the next study participants were asked to provide absolute evaluations of the unchanged, brighter, and darker versions of IAPS pictures.

\section{Study 4: Picture Brightness Influences Absolute Evaluations}


Instead of the relative judgments collected in Study 3, in the current study participants were asked to provide absolute evaluations of the unchanged, brighter, and darker IAPS pictures used in Study 3. Participants were told that for methodological reasons, they would be required to evaluate the pictures several times. It was not mentioned that the pictures in the three blocks were not identical. The IAPS pictures in each block differed in brightness, and consisted of either the original pictures, or brighter, or darker versions of the same pictures. The hypothesis was that picture brightness would influence evaluations even when participants were asked to perform absolute judgments.

Method.

Participants. A total of 84 participants (52 men, mean age 23, 76\% students) were approached through a participant database of the Eindhoven University of Technology. They completed the study in return for a monetary compensation of 1 euro.

Procedure. Participants were seated in individual cubicles and performed the evaluations behind a computer. The cover story and the stimuli were the same as in Study 3. The 10 IAPS pictures (numbers 7010, 7003, 7004, 7000, 7041, 6150, 7185, 7035, 7045, 7059) in each block were randomly presented, and the order of the three brightness blocks was counterbalanced across participants. Pictures appeared on the screen for $1000 \mathrm{~ms}$, after which a horizontal line appeared consisting of a 100 point scale, anchored with 'very negative’ on the left (scored as 0) and 'very positive' on the right (scored as 100). Participants could mark the line using the mouse, and the next picture appeared after clicking the 'continue' key.

Results. The evaluations of the neutral stimuli were averaged across the 10 pictures in each brightness condition. A repeated measures analyses with brightness block as the only within subjects factor revealed that the evaluations of the pictures differed reliably between the different brightness blocks, with darker pictures being rated least positive $(M=49.95, S D=$ 8.81), followed by the normal IAPS pictures $(M=51.90, S D=8.14)$, with brighter pictures 
being rated most positive $(M=52.84, S D=9.14), F(2,166)=6.26, p=.002, \mu_{p}^{2}=.07$.

Planned contrasts revealed that darker pictures were less positive than normal IAPS pictures, $F(1,83)=5.72, p=.02, \mu_{p}^{2}=.06$, but that the difference between normal IAPS pictures and brighter images was not pronounced enough to reach statistical significance, $F(1,83)=1.60, p$ $=.21$, n.s..

As we saw in Study 1, the brightness and contrast in IAPS pictures are correlated. Furthermore, the brightness of the pictures used in Study 3 and 4 were changed in Photoshop with the default algorithm that does not apply a linear brightness change over all pixels (which would result in quite noticable changes in hue and saturation), but applies proportional (nonlinear) adjustments to the picture, which adhere more closely to how humans perceive a change in brightness. As a consequence, contrast is a possible confound when examining the effects of a change in brightness on the evaluation of pictures. We analyzed the relation between the difference in brightness, contrast, and evaluation of the 20 manipulated pictures (10 brighter, 10 darker) compared to the original (unchanged) pictures in an analysis over items. As a consequence of the way Photoshop manipulates brightness, the change in brightness of these 20 stimuli was indeed correlated with a change in contrast $(r=.884, p<$ .001). In line with the results presented above, the brightness difference correlated with the change in evaluations $(r=.685, p<.001)$, but the change in contrast correlated with the change in evaluations as well $(r=.572, p=.008)$. Importantly however, when controlling for the change in contrast, the partial correlation between brightness and evaluation remained significant ( $r=.468, p=.043$ ), while the partial correlation between the change in contrast and evaluation was no longer significant when controlling for the change in brightness $(r=-.099, p$ $=$.687). This analysis provides support for our hypothesis that the change in brightness, and not the change in contrast, changes the evaluations of pictures. 
Discussion. The current results provide support for the hypothesis that the brightness of affective pictures influences their evaluations. When neutral IAPS images were made darker, they were evaluated more negatively. Whereas brighter IAPS pictures were judged as the more positive stimuli in the forced choice paradigm used in Study 3, increasing the brightness of neutral stimuli did not lead to more positive evaluations in the current study. These findings mirror earlier observations in Study 1, where the brightness of images in the positive and neutral subsets of the GAPED and IAPS differed significantly from negative pictures, but not from each other. It seems negativity is more strongly associated with darkness, than positivity is associated with brightness (see also Lakens et al., 2012). Although the size of the effect is modest, one should keep in mind that participants repeatedly evaluated the same stimuli, and that a tendency towards consistency over the ratings should be expected. The analysis over items supports our hypothesis that a change in brightness, and not a change in contrast, underlies the change in evaluations of the maniputlated pictures.

\section{Study 5: Light and Dark Stimuli as Primes in an Affective Priming Task}

The previous two experiments established that the brightness of IAPS pictures can influence their explicit evaluations. However, IAPS pictures are just as often used in research on implicit attitudes and automatic evaluations. For example, affective priming procedures, where affective pictures are presented briefly before participants respond to positive or negative target stimuli, have often relied on IAPS pictures as primes (e.g., Degner \& Wentura, 2010; Hermans, De Houwer \& Eelen, 1994). Given that the brightness of pictures influences how these pictures are explicitly evaluated (Studies 3 and 4), combined with the fact that affective pictures are often used in implicit measures, it is interesting to examine whether brightness differences between IAPS pictures used as primes can influence categorization times in an affective priming task. 
Participants. Seventy-nine students at Eindhoven University of Technology participated in the experiment in return for a small monetary compensation of 2 euro.

Design, materials and procedure. The priming task conformed to a 2 (prime brightness: bright vs. dark) x 2 (target word valence: positive vs. negative) within-subjects design. Target stimuli consisted of twenty positive and negative target words that were selected from the stimuli used by Meier and colleagues (2004; see also Lakens, 2012). They were presented in light grey (brightness value: 192). The two prime stimuli were constructed from a single, neutral IAPS picture, which depicts a floppy disk on a table (IAPS number 7003). This picture was digitally adjusted in Photoshop CS5, creating a brighter (brightness value: 149.51) and darker (brightness value: 27.02) version, which were used as primes (see Figure 4). An extreme brightness difference between both primes was chosen, to ensure that the brightness difference between primes was clearly visible against the grey background (RGB: 128; 128; 128).

The task started with 8 practice trials, followed by 80 experimental trials. Within each trial, the sequence of events was as follows: At the beginning of the trial, the prime was presented on the screen for $1000 \mathrm{~ms}$, immediately followed by the target word, which was presented until a response was registered. Following a response (either the 'z' or '/’ key, with the key assignment counterbalanced between participants), the next prime appeared immediately. The long prime presentation was chosen to minimize consecutive brightness changes during the task between fixation cues, primes, targets and empty screens. Results.

One participant was excluded from the analyses for excessive errors (more than $78 \%$ wrong responses). The analyses are performed on correct responses only (mean response time was 727 ms, mean error rate was 3\%). Following Klauer, Teige-Mocigemba, and Spruyt (2009) outliers were excluded from the analyses (5.9\% of the trials) according to Tukey’s 
criterion (response times 1.5 times the interquartile range below the first or above the third quartile).

Response latencies were submitted to a 2 (prime brightness) X 2 (target valence) repeated measures ANOVA. A significant prime brightness by target valence interaction, $F(1$, 77) $=16.02, p<.001, \eta_{\mathrm{p}}{ }^{2}=.17^{3}$, revealed that participants evaluated positive words more quickly after the bright $(M=671, S D=100)$ compared to dark $(M=685, S D=110)$ IAPS picture, whereas negative words were evaluated more quickly after a dark $(M=692, S D=$ 104) compared to a light $(M=704, S D=117)$ IAPS picture (see Figure 5$)$. Both the priming effect for positive $(M=14, S D=43)$ as for negative $(M=13, S D=43)$ words was significantly different from zero, $t(77)=2.89, p=.005$, and $t(77)=2.62, p=.011$, respectively. Submitting the errors to a 2 (prime brightness) X 2 (target valence) repeated measures ANOVA did not reveal a significant interaction, $F(1,77)=1.39, p=.225$. Discussion.

Affective pictures are often used as primes in affective priming tasks. Study 5 suggests that the brightness of affective pictures can influence the categorization times of positive and negative words. These results provide initial support for the idea that the brightness of naturalistic pictures can influence evaluations more automatically than in the explicit evaluations in Studies 3 and 4. These findings extend earlier studies that have revealed an automatic association between negativity and black, and positivity and white (Meier et al., 2004; Okubo \& Ishikawa, 2011).

These current findings should be interpreted with caution for several reasons. First of all, we only used two prime stimuli. This was done to increase the salience of the brightness differences. It therefore remains a question whether the observed effects of brightness differences on categorization times generalizes to affective priming tasks where a wider range of stimuli is used. Based on recent studies that have revealed how priming effects decrease or 
even disappear when the salience of the affective dimension is reduced (Everaert, Spruyt, \& De Houwer, 2011) it seems possible that the effect of brightness in evaluative priming tasks may decrease when the salience of brightness differences is reduced. Second, we modified the priming task to reduce brightness differences within each trial by removing the fixation point and the blank screen before and after the presentation of the prime and target. It is difficult to know how the change in timing routine (presenting the prime for $1000 \mathrm{~ms}$, and removing the intertrial interval, fixation cue, and any blank screens between the prime and target) has influenced reaction times, especially because this timing aspect of priming procedures has not been systematically examined (Wentura \& Degner, 2010). Future research should examine whether the findings observed here generalize to more traditional affective priming paradigms.

\section{General Discussion}

The current studies examined whether the evaluations of pictures were related to the brightness of these pictures. In Study 1 we found that positive pictures are brighter than negative pictures in two affective picture databases (the IAPS and the GAPED). Study 2 revealed that experimenters tend to select pictures for positive experimental conditions that on average are brighter than the pictures selected for negative experimental conditions. This brightness difference can be explained by the fact that researchers select affective pictures on the extremity of their affective rating, but do not control for brightness differences that exist within the affective picture databases. Going beyond correlational support for our hypothesis, Studies 3 and 4 showed that brighter versions of neutral pictures were evaluated more positively than darker versions of the same picture. Study 5 revealed that people categorized positive words more quickly than negative words after a bright prime picture, and vice versa for negative pictures. Together, these studies provide strong support for the notion that the 
valence of pictures is in part determined by their brightness, or a brightness bias in the evaluation of naturalistic photographs.

In Study 1 neutral GAPED images were equally bright as positive pictures, and in Study 4 IAPS pictures for which the brightness was not digitally adjusted were evaluated equally positive as pictures for which the brightness was digitally increased. These results provide preliminary indications that the association between darkness and negativity might be more pronounced than the association between positivity and brightness, in line with earlier research (Lakens et al., 2012). At the same time, when brightness differences are salient enough (e.g., when two pictures are presented in close temporal proximity such as in Study 3) brighter pictures are evaluated more positively, and Study 2 showed that researchers selected negative pictures that were significantly darker than neutral pictures, but positive pictures that were significantly brighter than neutral pictures.

Although the presence of an association between the brightness and valence of stimuli has been established in previous research (e.g., Adams \& Osgood, 1973), the relationship between brightness and valence in naturalistic photographs deserves a detailed examination because of the methodological implications on the one hand, and the great potential for practical applications on the other hand. On a methodological level, the current studies indicate an increased need to control for brightness differences between negative, neutral, and positive pictures in psychological research. We want to stress that the relationship between brightness and valence in affective picture databases is not problematic for most of the studies that have used these stimuli. After all, the evaluative ratings for these stimuli remain perfectly valid.

However, researchers should realize that the evaluative ratings of the pictures are not completely due to their content, but might also in part be based on their brightness. This means that the observed relationship between brightness and valence in the IAPS and GAPED could potentially be problematic if researchers have the specific hypothesis that the affective content 
of pictures causes the observed results. If research is explicitly aimed at examining the affective content of naturalistic pictures, we repeat the recommendation of Bradley and Lang (2007) to control for perceptual characteristics of naturalistic photographs, with a special focus on brightness differences. For example, research that relies on IAPS pictures to investigate affective impairments in psychiatric and neurological populations should either make sure that these populations do not differ from control participants in the way they process brightness differences, or control for brightness differences between positive and negative stimuli.

Note that although the recommendation the control for differences in picture brightness is good practice, a strong association between brightness and valence implies that modifying the brightness of stimuli might also change the valence rating of the stimulus (see Study 3 and 4). It is therefore important to collect new evaluations of IAPS pictures that are digitally modified (see for example Lavender \& Hommel, 2007). Alternatively, researchers might be able to select pictures that do not differ on brightness between conditions, although this might not be easy when stimuli from a specific thematic subset are needed (e.g., attacking humans and animals). To aid researchers to control for brightness differences, we have included a list with the average brightness and contrast values of all IAPS and GAPED pictures in the supplementary materials.

Besides methodological implications, the finding that darker images are evaluated more negatively has clear practical implications. Advertising agencies might do well to make sure visual media in commercials and advertisements are bright enough, given that dark pictures might lead to more negative product evaluations. It should be noted that the effects of picture brightness in Studies 3 and 4 were observed within participants, and whether such effects hold between participants is a question for further research. At the same time, people often see several images or videos in rapid succession (for example when watching advertisements on television or when flipping through the pages of a magazine). 
Although the current studies provide strong support for the presence of a valencebrightness association in naturalistic pictures, future research could shed more light on the nature of this relationship. Additional curve estimation analyses in Study 1 confirmed the assumption that within the IAPS and GAPED databases, the relationship between valence and brightness is best described as linear (instead of for quadratic, cubic, logarithmic, or exponential). However, it could be that a non-linear brightness-valence relationship might better describe some specific sets of pictures, such as when these picture sets include a large number of extremely bright or extremely dark pictures.

Several studies have examined the metaphorical relationship between the black-white opposition and the positive-negative opposition (Lakens et al., 2012; Meier, et al., 2004, Okubo \& Ishikawa, 2011). Examining whether valence is also related to more continuous brightness differences, Meier, Robinson, Crawford, and Ahlvers (2007) presented positive or negative words written in a grey font, after which participants were asked to select a grey square that best matched the brightness of the font. Participants were more likely to choose a lighter cube after a positive word than after a negative word. The current studies extend such insights to naturalistic images, but there might be important differences in the mechanisms underlying the relationship between brightness differences and valence differences on the one hand, and the 'white is positive and black is negative' relationship on the other hand (see Lakens et al., 2012).

Studies 3 and 4 revealed that manipulating the brightness of pictures can influence their evaluations, but these studies only used neutral pictures as stimuli. Consequently, it remains an empirical question whether brightness differences can influence the affective ratings of extremely affective pictures. Although one might expect smaller effects for pictures with strong affective content, it is also possible that a brightness-valence incongruency (i.e., a bright negative picture or a dark positive picture) has a strong effect on evaluations, and therefore this 
research question seems to be an interesting avenue for future research. Finally, we have not determined boundary conditions of the brightness-valence relationship. It might seem reasonable that an increase in brightness will reduce the positive evaluation of a picture, if it mirrors overexposure. So far however, this remains an empirical question.

With the current research, we hope to convince researchers of the importance to control for brightness differences when using affective pictures, and provide them with the necessary information to do so. At the same time, we believe these insights can be easily applied whenever visual media are used, given the potential of brightness manipulations to increase the positivity or negativity of the content that is communicated. 


\section{References}

Ackerman, J. M., Nocera, C. A., \& Bargh, J. A. (2010) Incedental haptic decisions influence social judgments and decisions, Science, 328, 1712-1715.

Adams, F. M., \& Osgood, C. E. (1973). A cross-cultural study of the affective meanings of color. Journal of Cross-Cultural Psychology, 4, 135-156.

* Amodio, D. M., \& Harmon-Jones, E. (2011). Trait emotions and affective modulation of the startle eyeblink: on the unique relationship of trait anger. Emotion, 11, 47-51. doi: $10.1037 / \mathrm{a} 0021238$

* Aue, T., Flykt A., \& Scherer K. R. (2007). First evidence for differential and sequential efferent effects of stimulus relevance and goal conduciveness appraisal. Biological Psychology, 74, 347-357. doi:10.1016/j.biopsycho.2006.09.001

* Azevedo, T. M., Volchan, E., Imbiriba, L. A., Rodrigues, E. C., Oliveira, J. M., Oliveira, L. F., et al., (2005). A freezing-like posture to pictures of mutilation. Psychophysiology, 42, 255-260. doi: 10.1111/j.1469-8986.2005.00287.x

* Bailey, K., West, R., \& Anderson, C. A. (2011). The association between chronic exposure to video game violence and affective picture processing: an ERP study. Cognitive, Affective, \& Behavioral Neuroscience, 11, 259-276. Doi: 10.3758/s13415-011-0029-y

Barsalou, L.W. (1999). Perceptual symbol systems. Behavioral and Brain Sciences, 22, 577609.

Bradley, M. M., Hamby, S., Low, A., \& Lang, P. J. (2007). Brain potentials in perception: Picture complexity and emotional arousal. Psychophysiology, 44, 364-373.

Bradley, M. M., \& Lang, P. J. (2007). The International Affective Picture System (IAPS) in the study of emotion and attention. In J. A. Coan and J. J. B. Allen (Eds.), Handbook of Emotion Elicitation and Assessment (pp. 29-46). New York: Cambridge University Press. 
* Baumgartner, T., Esslen, M., \& Jäncke, L. (2006). From emotion perception to emotion experience: Emotions evoked by pictures and classical music. International Journal of Psychophysiology, 60, 34-43. doi:10.1016/j.ijpsycho.2005.04.007

* Bernat, E., Patrick, C. J., Benning, S. D., \& Tellegen, A. (2006). Effects of picture content and intensity on affective physiological response. Psychophysiology, 43, 93-103.

* Buchholz, K., Schächinger, H., Wagner, M., Schorr, U., Sharma, A. M., \& Deter, H. C. (2001). Enhanced affective startle modulation in salt-sensitive subjects. Hypertension, 38, 1325-1329. doi: 10.1161/hy1101.096055

Calvo, M. G., \& Nummenmaa, L. (2007). Processing of unattended emotional visual scenes. Journal of Experimental Psychology: General, 136, 347-369.

* Carmen Pastor, M., Moltó, J., Vila, J., \& Lang, P. J. (2003). Startle reflex modulation, affective ratings and autonomic reactivity in incarcerated Spanish psychopaths. Psychophysiology, 40, 934-938. doi: 10.1111/1469-8986.00111

Chandler, J., Reinhard, D. \& Schwarz, N. (2012). To judge a book by its weight you need to know its content: Knowledge moderates the use of embodied cues. Journal of Experimental Social Psychology, 48, 948-952. doi: 10.1016/j.jesp.2012.03.003

* Cohen, A. S., Minor, K. S., Najolia, G. M., \& Hong, S. L. (2009). A laboratory-based procedure for measuring emotional expression from natural speech. Behavior research methods, 41, 204-212. doi:10.3758/BRM.41.1.204

* Cuthbert, B. N., Schupp, H. T., Bradley, M. M., Birbaumer, N., \& Lang, P. J. (2000). Brain potentials in affective picture processing: Covariation with autonomic arousal and affective report. Biological psychology, 52, 95-111.

Dan-Glauser, E. S., \& Scherer, K. R. (2011). The Geneva affective picture database (GAPED): a new 730-picture database focusing on valence and normative 
significance. Behavior Research Methods, 43, 468-477. doi: 10.3758/s13428-0110064-1

* D'Argembeau, A., \& Van der Linden, M. (2005). Influence of emotion on memory for temporal information. Emotion, 5, 503-507.

De Cesarei, A., \& Codispoti, M. (2006). When does size not matter? Effects of stimulus size on affective modulation. Psychophysiology, 43, 207-15.

Degner, J., \& Wentura, D. (2010). Automatic prejudice in childhood and early adolescence. Journal of Personality and Social Psychology, 98, 356-374.

* De Houwer, J., \& De Bruycker, E. (2007). Implicit attitudes towards meat and vegetables in vegetarians and nonvegetarians. International Journal of Psychology, 42, 158-165. doi: $10.1080 / 00207590601067060$

Delplanque, S., N'diaye, K., Scherer, K. R., \& Grandjean, D. (2007). Spatial frequencies or emotional effects? A systematic measure of spatial frequencies for IAPS pictures by a discrete wavelet analysis. Journal of Neuroscience Methods, 165, 144-50.

* Drago, V., Foster, P. S., Chanei, L., Rembisz, J., Meador, K., Finney, G., \& Heilman, K. M. (2010). Emotional indifference in Alzheimer's disease. The Journal of Neuropsychiatry and Clinical Neurosciences, 22, 236-242.

Elliot, A.J., \& Maier, M.A. (2007). Color and Psychological Functioning, Current Directions in Psychological Science, 16, 250-254.

Elliot, A. J., \& Maier, M. A. (2012). Color-in-Context Theory. In P. Devine, \& A. Plant (Eds.), Advances in Experimental Social Psychology (Vol. 45, pp. 61-125). Burlington: Academic Press.

Elliot, A. J., \& Niesta, D. (2008). Romantic red: Red enhances men's attraction to women. Journal of Personality and Social Psychology, 95, 1150-1164. 
Elliot, A. J. \& Niesta Kayser, D. Greitemeyer, T., Lichtenfeld, S., Gramzow, R. H., Maier, M. A., \& Liu, H. (2010). Red, Rank, and Romance in Women Viewing Men. Journal of Experimental Psychology: General, 139, 399-417.

* Everaert, T., Spruyt, A., \& De Houwer, J. (2011). On the (un)conditionality of automatic attitude activation: The valence proportion effect. Canadian Journal of Experimental Psychology, 65, 125-132. doi: 10.1037/a0022316.

* Field, A. P., \& Moore, A. C. (2005). Dissociating the effects of attention and contingency awareness on evaluative conditioning effects in the visual paradigm. Cognition \& Emotion, 19, 217-243.

* Fockenberg, D. A., Koole, S. L., \& Semin, G. R. (2006). Backward affective priming: Even when the prime is late, people still evaluate. Journal of Experimental Social Psychology, 42, 799-806.

* Friederich, H. C., Kumari, V., Uher, R., Riga, M., Schmidt, U., Campbell, I. C., et al. (2006). Differential motivational responses to food and pleasurable cues in anorexia and bulimia nervosa: a startle reflex paradigm. Psychological Medicine, 36, 1327-1335. doi:10.1017/S0033291706008129

Gawronski, B., Deutsch, R., \& Seidel, O. (2005). Contextual influences on implicit evaluation: A test of additive versus contrastive effects of evaluative context stimuli in affective priming. Personality and Social Psychology Bulletin, 31, 1226-1236.

Grillon, C., Pellowski, M., Merikangas, K. R., \& Davis, M. (1997). Darkness facilitates acoustic startle reflex in humans. Biological Psychiatry, 42, 453-460.

Guilford, J. P. (1934) The Affective Value of Color as a Function of Hue, Tint, and Chroma. Journal of Experimental Psychology, 17,342-370.

* Hajcak, G., \& Olvet, D. M. (2008). The persistence of attention to emotion: brain potentials during and after picture presentation. Emotion, 8, 250-255. doi: 10.1037/15283542.8.2.250 
* Hajcak, G., \& Dennis, T. A. (2009). Brain potentials during affective picture processing in children. Biological psychology, 80, 333-338. doi:10.1016/j.biopsycho.2008.11.006.

Hermans, D., De Houwer, J., \& Eelen, P. (1994). The affective priming effect: Automatic activation of evaluative information in memory. Cognition and Emotion, 8, 515-533.

* Hess, U., Sabourin, G., \& Kleck, R. E. (2007). Postauricular and eyeblink startle responses to facial expressions. Psychophysiology, 44, 431-435. doi: 10.1111/j.14698986.2007.00516.x

* Hillman, C. H., Rosengren, K. S., \& Smith, D. P. (2004). Emotion and motivated behavior: postural adjustments to affective picture viewing. Biological Psychology, 66, 51-62. doi:10.1016/j.biopsycho.2003.07.005

* Hindi Attar, C., Andersen, S. K., \& Müller, M. M. (2010). Time course of affective bias in visual attention: Convergent evidence from steady-state visual evoked potentials and behavioral data. Neuroimage, 53, 1326-1333. doi:10.1016/j.neuroimage.2010.06.074.

* Huijding, J., \& De Jong, P. J. (2005). A pictorial version of the Extrinsic Affective Simon Task: sensitivity to generally affective and phobia-relevant stimuli in high and low spider fearful individuals. Experimental Psychology, 52, 289. doi: 10.1027/16183169.52.4.289

Humphrey, N. K. (1971). Color and brightness preferences in monkeys. Nature, 229, 615617.

* Keil, A., Bradley, M. M., Hauk, O., Rockstroh, B., Elbert, T., \& Lang, P. J. (2002). Large-scale neural correlates of affective picture processing. Psychophysiology, 39, 641-649. doi: 10.1017.S0048577202394162

* Kisley, M. A., Wood, S., \& Burrows, C. L. (2007). Looking at the Sunny Side of Life AgeRelated Change in an Event-Related Potential Measure of the Negativity Bias. Psychological Science, 18, 838-843. 
Klauer, K. C., \& Musch, J. (2003). Affective priming: Findings and theories. In J. Musch \& K. C. Klauer (Eds.), The psychology of evaluation: Affective processes in cognition and emotion (pp. 7-49). Mahwah, NJ: Lawrence Erlbaum.

Klauer, K. C., Teige-Mocigemba, S., \& Spruyt, A. (2009). Contrast effects in spontaneous evaluations: A psychophysical account. Journal of Personality and Social Psychology, 96, 265-287.

* Kronshage, U., Kroener-Herwig, B., \& Pfingsten, M. (2001). Kinesiophobia in chronic low back pain patients—does the startle paradigm support the hypothesis?. International Journal of Behavioral Medicine, 8, 304-318.

* Kuniecki, M., Urbanik, A., Sobiecka, B., Kozub, J., \& Binder, M. (2002). Central control of heart rate changes during visual affective processing as revealed by fMRI. Acta neurobiologiae experimentalis, 63, 39-48.

Lakens, D. (2012). Polarity Correspondence in Metaphor Congruency Effects: Structural Overlap Predicts Categorization Times for Bi-Polar Concepts Presented in Vertical Space. Journal of Experimental Psychology: Learning, Memory, and Cognition, 38, 726-723. doi: 10.1037/a0024955.

Lakens, D., Semin, G. R., \& Foroni, F. (2012). But for the bad, there would not be good: Grounding valence in brightness through structural similarity. Journal of Experimental Psychology: General, 141, 584-594. doi: 10.1037/a0026468.

Lang, P.J., Bradley, M.M., \& Cuthbert, B.N. (2008). International affective picture system (IAPS): Affective ratings of pictures and instruction manual. Technical Report A-8. University of Florida, Gainesville, FL.

* Lang, P. J., Bradley, M. M., Fitzsimmons, J. R., Cuthbert, B. N., Scott, J. D., Moulder, B., \& Nangia, V. (1998). Emotional arousal and activation of the visual cortex: an fMRI analysis. Psychophysiology, 35, 199-210. 
* Langeslag, S. J., \& van Strien, J. W. (2009). Aging and emotional memory: The co-occurrence of neurophysiological and behavioral positivity effects. Emotion, 9, 369-377.

* Larson, C. L., Nitschke, J. B., \& Davidson, R. J. (2007). Common and distinct patterns of affective response in dimensions of anxiety and depression. Emotion, 7, 182-191. doi: 10.1037/1528-3542.7.1.182

Lavender, T., \& Hommel, B. (2007). Affect and action: Towards an event-coding account. Cognition and Emotion, 21, 1270-1296.

* Liu, D. L. J., Graham, S., \& Zorawski, M. (2008). Enhanced selective memory consolidation following post-learning pleasant and aversive arousal. Neurobiology of learning and memory, 89, 36-46. doi:10.1016/j.nlm.2007.09.001

Maglio, S. J. \& Trope, Y. (2012): Disembodiment: Abstract construal attenuates the influence of contextual bodily state in judgment. Journal of Experimental Psychology: General, 141, 211-216. doi: 10.1037/a0024520

Meier, B. P., Robinson, M. D., \& Clore, G. L. (2004). Why good guys wear white: Automatic inferences about stimulus valence based on brightness. Psychological Science, 15, 82-87.

Meier, B.P., Robinson, M.D., Crawford, L.E., \& Ahlvers, W.J. (2007). When 'light' and ‘dark’ thoughts become light and dark responses: Affect biases brightness judgments. Emotion, 7, 366-376.

* Meinhardt, J., \& Pekrun, R. (2003). Attentional resource allocation to emotional events: An ERP study. Cognition \& Emotion, 17, 477-500. doi: 10.1080/02699930244000039

* Mikels, J. A., Fredrickson, B. L., Larkin, G. R., Lindberg, C. M., Maglio, S. J., \& ReuterLorenz, P. A. (2005). Emotional category data on images from the International Affective Picture System. Behavior research methods, 37, 626-630.

Mitchell, A. A. (1986). The effect of verbal and visual components of advertisements on brand attitudes and attitude toward the advertisement. Journal of Consumer Research, $13,12-24$. 
* Mühlberger, A., Neumann, R., Wieser, M. J., \& Pauli, P. (2008). The impact of changes in spatial distance on emotional responses. Emotion, 8, 192-198. doi: 10.1037/15283542.8.2.192

* Müller, M. M., Keil, A., Gruber, T., \& Elbert, T. (1999). Processing of affective pictures modulates right-hemispheric gamma band EEG activity. Clinical Neurophysiology, 110, 1913-1920.

Okubo, M. \& Ishikawa, K. (2011). Automatic semantic association between emotional valence and brightness in the right hemisphere. Cognition and Emotion, 25, 12731280. doi:10.1080/02699931.2010.541658

Osgood, C.E., Suci, G., \& Tannenbaum, P. (1957). The measurement of meaning. Urbana, IL: University of Illinois Press.

* Payne, B. K., Cheng, C. M., Govorun, O., \& Stewart, B. D. (2005). An inkblot for attitudes: affect misattribution as implicit measurement. Journal of Personality and Social Psychology, 89, 277. doi: 10.1037/0022-3514.89.3.277

Pazda, A. D., Elliot, A. J., \& Greitemeyer, T. (2012). Sexy red: Perceived sexual receptivity mediates the red-attraction relation in men viewing woman. Journal of Experimental Social Psychology, 48, 787-790.

Peli, E. (1990). Contrast in complex images. Journal of the Optical Society of America A, 7(10), 2032-2040, doi:10.1364/JOSAA.7.002032

* Pleyers, G., Corneille, O., Luminet, O., \& Yzerbyt, V. (2007). Aware and (dis) liking: itembased analyses reveal that valence acquisition via evaluative conditioning emerges only when there is contingency awareness. Journal of Experimental Psychology: Learning, Memory, and Cognition, 33, 130-144. doi: 10.1037/0278-7393.33.1.130 
* Quirin, M., Kazén, M., \& Kuhl, J. (2009). When nonsense sounds happy or helpless: The Implicit Positive and Negative Affect Test (IPANAT). Journal of Personality and Social Psychology, 97, 500-516. doi: 10.1037/a0016063

* Robinson, M. D., Storbeck, J., Meier, B. P., \& Kirkeby, B. S. (2004). Watch out! That could be dangerous: Valence-arousal interactions in evaluative processing. Personality and Social Psychology Bulletin, 30, 1472-1484. doi: 10.1177/0146167204266647

* Ruys, K. I., \& Stapel, D. A. (2009). Learning to like or dislike by association: No need for contingency awareness. Journal of Experimental Social Psychology, 45, 1277-1280. doi: 10.1016/j.jesp.2009.06.012

* Sandt, A. R., Sloan, D. M., \& Johnson, K. J. (2009). Measuring appetitive responding with the postauricular reflex. Psychophysiology, 46, 491-497. doi: 10.1111/j.14698986.2009.00797.x

Sherman, G. D., \& Clore, G. L. (2009). The color of sin: White and black are perceptual symbols of moral purity and pollution. Psychological Science, 20, 1019-1025.

* Shah, S. G., Klumpp, H., Angstadt, M., Nathan, P. J., \& Phan, K. L. (2009). Amygdala and insula response to emotional images in patients with generalized social anxiety disorder. Journal of Psychiatry \& Neuroscience, 34, 296.

* Sierra, M., Senior, C., Dalton, J., McDonough, M., Bond, A., Phillips, M. L., et al. (2002). Autonomic response in depersonalization disorder. Archives of General Psychiatry, 59, 833-838.

* Smith, N. K., Cacioppo, J. T., Larsen, J. T., \& Chartrand, T. L. (2003). May I have your attention, please: Electrocortical responses to positive and negative stimuli. Neuropsychologia, 41, 171-183. 
*Sterzer P., Stadler C., Krebs A., Kleinschmidt A., \& Poustka F. (2005). Abnormal neural responses to emotional visual stimuli in adolescents with conduct disorder. Biological Psychiatry, 57, 7-15.

Stewart, K., McVeigh, M., Stojkov, I., \& Daubman, K. (2012). Heavy issues are more important. Retrieved November 15, 2012 from http://www.PsychFileDrawer.org/replication.php?attempt=MTMy

Valberg, A. (2005). Light Vision Color. Wiley, Chichester.

Valdez, P., \& Mehrabian, A. (1994). Effects of color on emotions. Journal of Experimental Psychology. General, 123, 394 - 409. DOI: 10.1037/0096-3445.123.4.394

Wentura, D., \& Degner, J. (2010). A practical guide to sequential priming and related tasks. In B. Gawronski \& B. K. Payne (Eds.), Handbook of implicit social cognition: Theory, measurement, and applications (pp. 95-116). New York, NY: Guilford.

* de Wied, M., \& Verbaten, M. N. (2001). Affective pictures processing, attention, and pain tolerance. Pain, 90, 163-172.

Wieser, M. J., Mühlberger, A., Alpers, G. W., Macht, M., Ellgring, H. \& Pauli, P. (2006). Emotion processing in Parkinson's disease: dissociation between early neuronal processing and explicit ratings. Clinical Neurophysiology, 117, 94-102.

Yartz, A. R., \& Hawk, L. W., Jr. (2002). Addressing the specificity of affective startle modulation: Fear versus disgust. Biological Psychology, 59, 55-68. 


\section{Footnotes}

${ }^{1}$ Note that several IAPS pictures have 2 sets of ratings in the 2008 Tech manual for pictures that were included in two separate rating studies: 1230, 1590, 1610, 1640, 1670, 2210, 3000, 3010, 4220, 4520, 6200, 9090. The first reported set of ratings was used in the current analysis, but both ratings yielded identical results.

${ }^{2}$ One study used only nine exceptionally bright positive pictures, one study used only 2 exceptionally bright neutral pictures, and one study used only 2 exceptionally bright negative pictures.

${ }^{3}$ Note that the interaction was also significant without removing outliers, $F(1,77)=5.39, p=$ $.023, \eta_{\mathrm{p}}{ }^{2}=.07$, or when using an a-priori determined cutoff value of $1000 \mathrm{~ms}$ (cf. Gawronski, Deutsch, \& Seidel, 2005), $F(1,77)=25.76, p<.001, \eta_{\mathrm{p}}{ }^{2}=.25$. 
Table 1.

Average brightness values (and SD) for subsets of the GAPED

\begin{tabular}{lccc}
\hline Category & $M$ & $(S D)$ & $N$ \\
\hline Animal mistreatment & 113.53 & 25.50 & 124 \\
Human concerns & 113.52 & 25.50 & 105 \\
Snakes & 103.82 & 28.01 & 133 \\
Spiders & 110.64 & 35.64 & 158 \\
Neutral & 126.98 & 28.74 & 89 \\
Positive & 119.72 & 36.33 & 121 \\
\hline
\end{tabular}


Figure 1. Scatterplots and linear regression lines for the relationship between the valence and brightness of IAPS and GAPED stimuli analyzed in Study 1.

\section{IAPS}

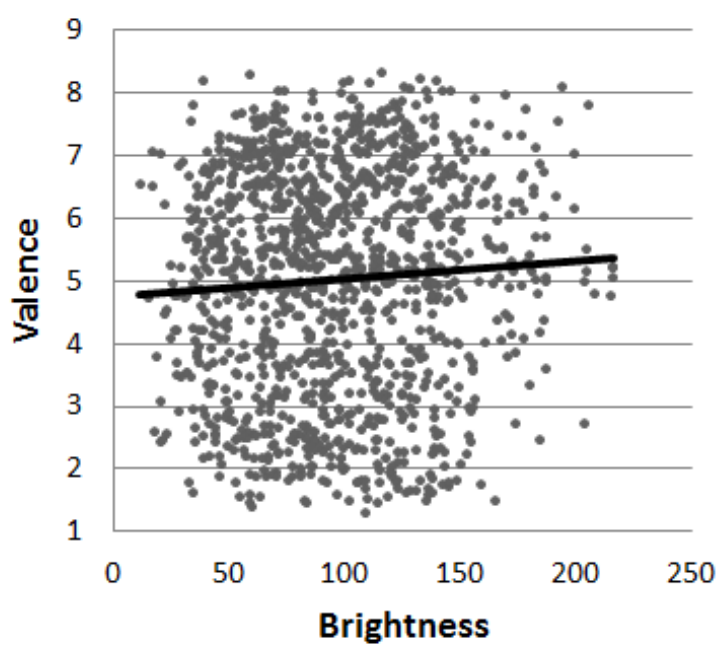

\section{GAPED}

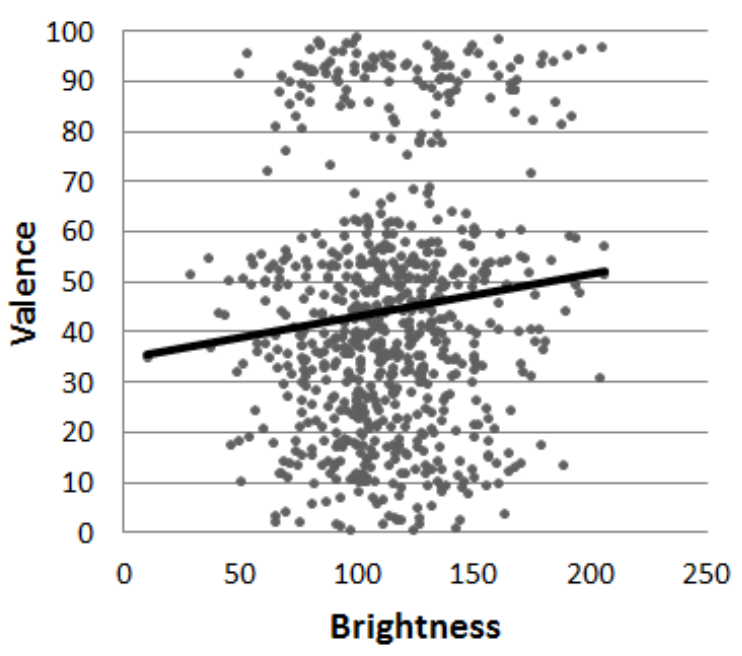


Figure 2. Average brightness of positive (left) neutral (middle) and negative (right) IAPS pictures analyzed in Study 2. 
Figure 3. Example of a darker (left), original (middle) and lighter (right) stimulus picture used in Study 3. This example is not identical to the IAPS stimulus due to copyright restrictions.




Figure 4. Example of the dark (left) and light (right) prime picture used in Study 5. This example is not identical to the IAPS stimulus due to copyright restrictions.

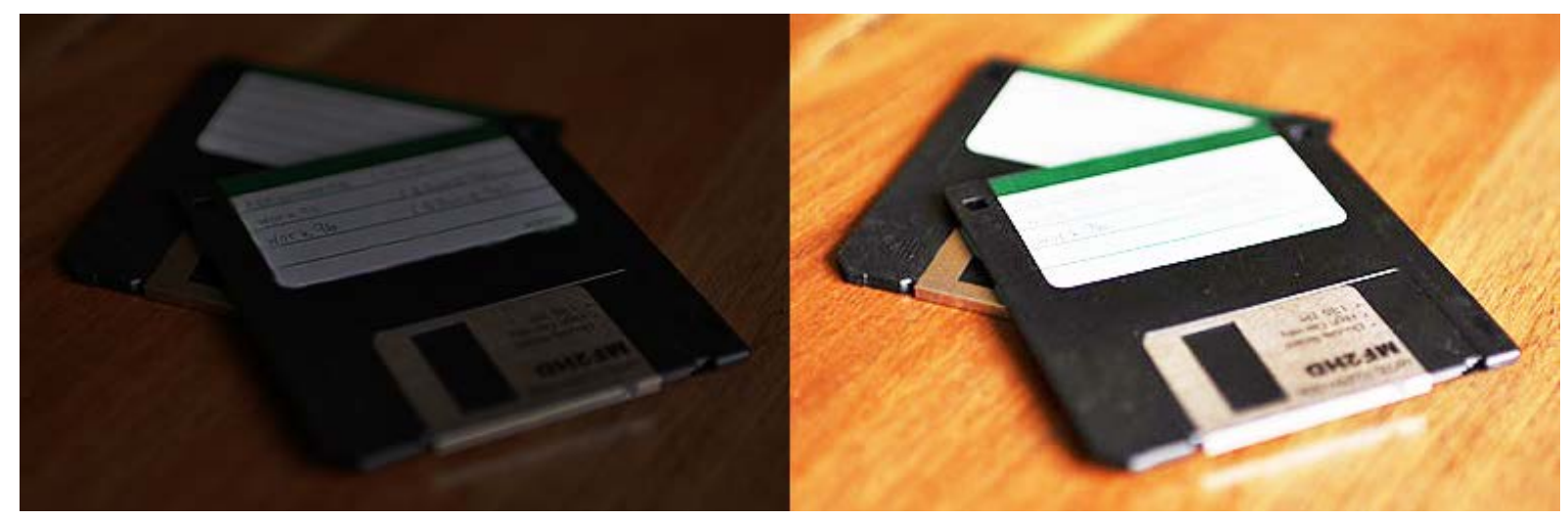


Figure 5. Average reaction times for positive and negative words as a function of a preceding light or dark prime in Study 5.

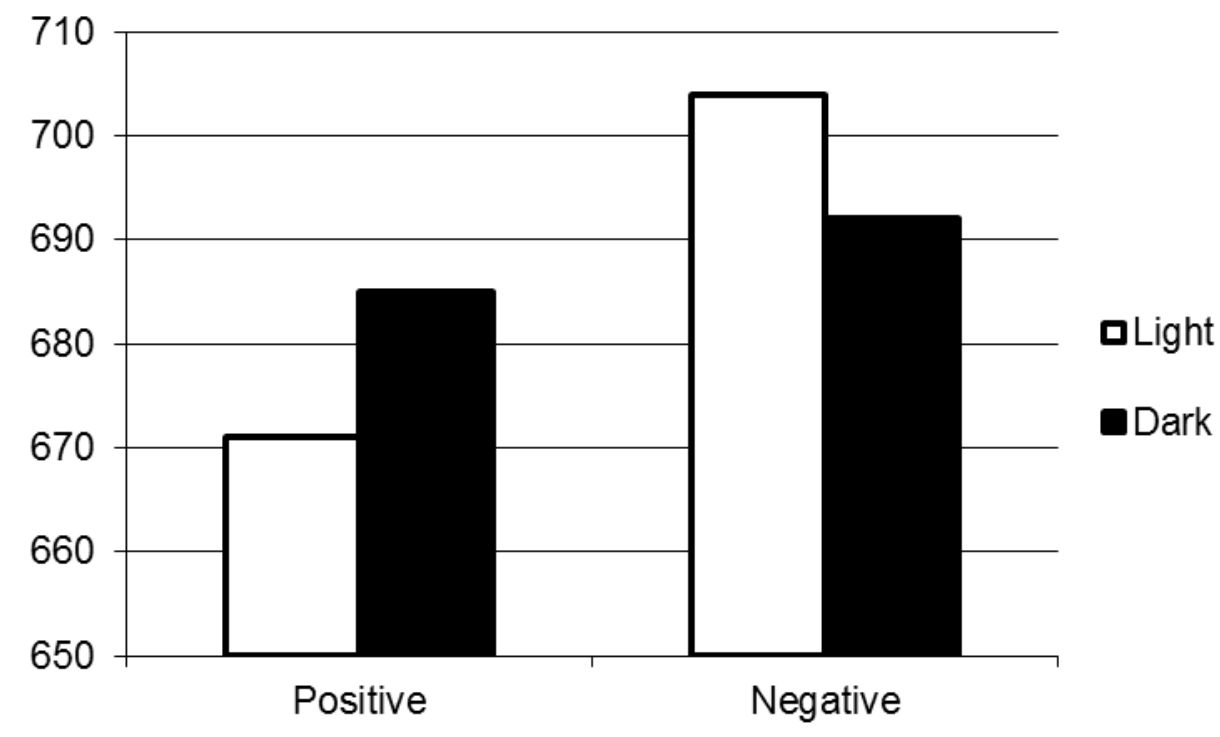




\section{Appendices}

Appendix A. Average brightness values (and SD) for subsets of the IAPS.

\begin{tabular}{|c|c|c|c|c|c|c|}
\hline & $\mathrm{N}$ & M & SD & $\mathrm{t}$ & $\mathrm{p}$ & Cohen's d \\
\hline ABSTRACT & 16 & 94.03 & 37.87 & -0.22 & 0.83 & \\
\hline ACCIDENT & 37 & 96.09 & 32.57 & -0.01 & 1.00 & \\
\hline ANIMAL_CRUELTY & 16 & 120.35 & 30.31 & 3.20 & 0.01 & 0.78 \\
\hline ATTACKING_HUMANS_OR_ANIMALS & 92 & 78.66 & 37.01 & -4.53 & 0.00 & 0.47 \\
\hline SOCIAL & 149 & 91.61 & 38.39 & -1.44 & 0.15 & \\
\hline CITY & 27 & 98.89 & 44.11 & 0.32 & 0.75 & \\
\hline FAMILY & 32 & 96.85 & 41.43 & 0.10 & 0.92 & \\
\hline DOMESTIC_ANIMAL & 13 & 106.66 & 31.65 & 1.20 & 0.25 & \\
\hline SPIDER & 7 & 88.43 & 51.69 & -0.40 & 0.71 & \\
\hline EROTIC & 89 & 94.58 & 43.90 & -0.33 & 0.74 & \\
\hline FOOD & 54 & 99.90 & 43.57 & 0.64 & 0.53 & \\
\hline HOUSEHOLD_OBJECTS & 79 & 119.69 & 47.93 & 4.37 & 0.00 & 0.49 \\
\hline ILLNESS_DIRT & 35 & 102.99 & 28.75 & 1.41 & 0.17 & \\
\hline MEDICAL & 27 & 109.40 & 38.57 & 1.79 & 0.09 & \\
\hline MEN & 62 & 82.14 & 37.92 & -2.91 & 0.01 & 0.37 \\
\hline MUTILATIONS & 74 & 95.33 & 32.97 & -0.21 & 0.84 & \\
\hline NATURE & 69 & 97.92 & 34.62 & 0.43 & 0.67 & \\
\hline OTHER & 44 & 101.83 & 35.83 & 1.05 & 0.30 & \\
\hline ROMANCE & 29 & 89.96 & 36.75 & -0.91 & 0.37 & \\
\hline SOCIAL_DEVIANCE & 18 & 74.91 & 38.93 & -2.31 & 0.03 & 0.55 \\
\hline SPORTS & 57 & 109.92 & 39.24 & 2.65 & 0.01 & 0.35 \\
\hline TECHNOLOGY & 26 & 98.56 & 34.07 & 0.36 & 0.72 & \\
\hline WILDLIFE & 50 & 98.52 & 40.92 & 0.41 & 0.68 & \\
\hline WOMEN & 26 & 90.34 & 40.38 & -0.73 & 0.47 & \\
\hline ADVENTURE & 27 & 103.60 & 34.50 & 1.13 & 0.27 & \\
\hline SNAKE & 19 & 68.50 & 30.81 & -3.91 & 0.00 & 0.90 \\
\hline MUSHROOM & 8 & 76.73 & 19.24 & -2.85 & 0.03 & 1.01 \\
\hline Total & 1182 & 96.13 & 39.78 & & & \\
\hline
\end{tabular}

Category label, number of stimuli in each category $(N)$, average brightness value $(M)$ and standard deviation (SD) for pictures in each category, $t$-value for a one-sample $t$-test against the average brightness value of the entire IAPS database, with the associated $p$-values and (when significant) effect size. 
Appendix B. Average brightness values for positive, negative, and neutral pictures used in 47 published studies.

\begin{tabular}{llll}
\hline 1st Author & Positive & Negative & Neutral \\
\hline Amodio2010 & 114,08 & 91,61 & 93,55 \\
Aue2006 & & 77,62 & 87,64 \\
Azevedo2005 & 146,86 & 99,35 & 101,34 \\
Bailey2011 & 107,75 & 83,41 & 82,69 \\
Baumgartner2006 & 107,96 & 81,79 & \\
Bernat2006 & 94,50 & 70,57 & 90,24 \\
Buchholz2001 & 97,60 & 73,91 & 85,74 \\
CarmenPastor2003 & 96,97 & 79,48 & 100,33 \\
Cohen2009 & 92,72 & 92,17 & \\
Coombes2006 & 105,17 & 106,80 & 44,88 \\
Cuthbert2000 & 123,64 & 88,35 & 88,02 \\
Dargembeau2005 & 94,96 & 96,94 & 81,58 \\
DeHouwer2007 & 119,51 & 91,77 & \\
Drago2010 & 92,20 & 82,53 & \\
Evereart2011 & 103,93 & 87,36 & \\
Field2005 & 127,86 & 146,71 & 98,09 \\
Fockenberg2008 & 104,64 & 75,02 & 93,14 \\
Friederich2006 & 104,09 & 82,37 & 108,42 \\
Hajcak2008 & 101,87 & 92,57 & 94,09 \\
Hajcak2009 & 91,22 & 77,42 & 94,51 \\
Hess2007 & 107,23 & 120,60 & \\
Hillman2004 & 101,37 & 86,73 & \\
HindiAttar2010 & 100,21 & 90,12 & 91,75 \\
Huijding2005 & 96,12 & 100,60 & \\
Keil2002 & 90,79 & 85,03 & \\
Kisley2007 & 94,39 & 108,70 & 96,61 \\
Kroonshage2001 & 80,90 & 95,61 & \\
Kuniecki2003 & 90,70 & 87,63 & \\
Lang1998 & 97,39 & 87,10 & \\
Langeslag2009 & 92,16 & 94,13 & \\
Larson2010 & 104,78 & 91,22 & 96,92 \\
Lui2008 & 97,54 & 84,41 & 80,67 \\
Meinhardt2003 & 110,67 & 92,70 & 103,30 \\
Mikels2005 & 97,60 & 86,20 & \\
Muhlberger2008 & 145,39 & 62,12 & 89,24 \\
Muller1999 & 90,79 & 82,80 & 70,61 \\
Payne2005 & 105,12 & 89,69 & \\
Pleyers2007 & 106,27 & 54,10 & \\
Quirin2009 & 95,97 & 71,29 & 96,32 \\
Robinson2004 & 99,70 & 86,01 & \\
Ruys2009 & 126,08 & 83,62 & \\
Sandt2009 & 102,55 & 90,98 & 95,50 \\
Shah2008 & 102,32 & 91,80 & \\
Sierra2002 & 105,67 & 87,86 & 117,21 \\
Smith2003 & 102,13 & 100,90 & \\
Sterzer2004 & & 86,81 & 78,91 \\
Wied2001 & 102,80 & 84,88 & 88,33 \\
\hline \hline
\end{tabular}


Appendix C. Number of times negative (black) and positive (white) stimuli were selected (vertical axis) across the 47 experiments in Study 2 as a function of their valence (horizontal axis).

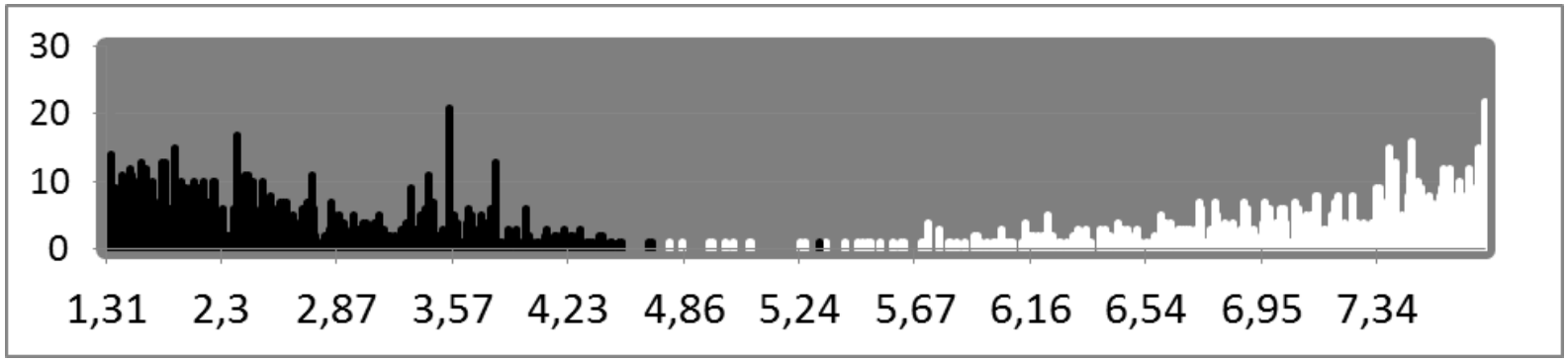

